\title{
Multimodal Imaging, OCT En Face, and OCT Angiography of an Anomalous Retinal Artery: Case Report and Review of the Literature
}

\author{
Beatriz Vidal-Villegas ${ }^{a}$ b Juan Antonio Miralles de Imperial-Ollero ${ }^{b}$, \\ Enrique Santos Bueso ${ }^{a}$, b Julián García-Feijoo ${ }^{a, b}$ \\ Maria Paz Villegas-Pérez ${ }^{b, c}$ \\ aOphthalmology Service, Hospital Clínico San Carlos, and Instituto de Investigación \\ Sanitaria del Hospital Clínico San Carlos (IdISSC), Universidad Complutense, Madrid, Spain; \\ ${ }^{b}$ Red Temática de Investigación Cooperativa OftaRed, Madrid, Spain; 'Ophthalmology \\ Service, Hospital General Universitario Reina Sofía, Murcia, Spain
}

\section{Keywords}

Anomalous macular artery - Optic coherence tomography angiography · Papillomacular bundle $\cdot$ Retinal nerve fiber layer raphe

\section{Abstract}

The purpose is to study for the first time the vascular plexuses and the retinal nerve fiber layer and raphe of a patient with a very uncommon anatomical variation: an anomalous retinal artery supplying the whole macula. We used multimodal imaging, en face spectral-domain optic coherence tomography, and spectral-domain optic coherence tomography angiography. One patient presented in his left eye a very unusual anatomical variation of macular vascularization. A retinal artery deriving from the inferior temporal retinal artery irrigated the whole macula. The formation of the papillomacular bundle and the temporal raphe nerve fiber layer has been attributed to the earlier development of the central retina and to the existence of 2 distinct watershed zones. However, there are very uncommon anatomical variations of the retinal vasculature in which large retinal vessels cross the raphe and could influence the morphology and structure of the nerve fiber layer of the posterior pole. We review the literature on the subject and document for the first time an anomalous artery that irrigates the whole macula, normal thickness and morphology of the nerve fiber layer, and the temporal raphe. 


\section{Case Reports in Ophthalmology}

\section{Introduction}

The retinal vessels appear early in the development from the superficial layers of the optic disc and reach the retinal periphery before birth [1]. These vessels usually divide into 4 branches, 2 nasal and 2 temporal. In the temporal retina, 2 hemispheres with distinct watershed zones superior and inferior are established, although communications between the superior and inferior systems exist at the level of the capillary plexus [2].

The large retinal vessels run at the level and parallel to the nerve fiber layer, and the superior and inferior distribution of the large retinal vessels in the temporal retina allows the temporal nerve fibers of the optic nerve to run horizontally in the papillomacular bundle and to surround the macula to form a temporal raphe that is the basis of the horizontal meridian of the visual field $[3,4]$. It is believed that the division of the human retina in 2 hemispheres with different vascularization and nerve fiber layer may have protective visual effects in the case of vascular occlusive or optic nerve diseases [3].

The formation of the nerve fiber temporal raphe has been attributed to the earlier development of the central retina and also to the existence of the watershed zones [3, 4]. However, anatomical variations of the retinal vasculature have been described in which large retinal vessels arteries or veins cross the horizontal retinal raphe. The veins that cross the temporal raphe are usually aberrant dilated veins that have been categorized as arterio-venous communications type 1 and received the names of congenital retinal macrovessels or, more recently, retinal venous malformations [5-7]. Large arteries that cross the retinal raphe are less common and have been reported in $<1 \%$ of the individuals $[8,9]$. None of the previous studies on anatomical variations of the retinal vasculature crossing the retinal raphe have investigated the morphology of the temporal retinal nerve fiber layer and raphe. We present one very unusual case of an anomalous retinal artery that crosses the horizontal raphe and explore the retinal vascularization and retinal nerve fiber layer and raphe.

\section{Case Presentation}

We present a case report of a 63-year-old man who was being treated in our service of a conjunctival intraepithelial neoplasia of the left eye. His uncorrected visual acuity was 20/20 in both eyes, and his fundus examination was normal in both eyes. However, he showed in the left eye a retinal artery that exited from the inferotemporal artery, crossed the papillomacular bundle, and bifurcated to give 2 branches that supplied both the superior and inferior macula (Fig. 1A, C).

We used multimodal imaging and en face SD-OCT and SD-OCTA (Spectralis, Heidelberg) to explore the retinal structure and vascularization. The SD-OCT documented normal macular structure and normal thickness of the peripapillary nerve fiber layer (Fig. 1A-D). The en face SD-OCT documented that the normal linear morphology of the papillomacular bundle was not affected by the traversing artery because it ran underlying the nerve fiber layer (Fig. 1F) and that the morphology of the nerve fiber raphe was normal (Fig. 1E). The SD-OCTA documented that the 3 retinal vascular plexuses had a normal morphology, with a preserved foveal avascular zone (Fig. 1F-J).

\section{Discussion and Conclusion}

The retina presents a temporal horizontal raphe that divides the retina into 2 hemispheres: superior and inferior [4]. These hemispheres contain 2 different vascular systems that communicate only at the level of the 3 capillary plexuses, but in the parafovea

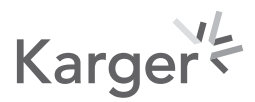




\section{Case Reports in Ophthalmology}
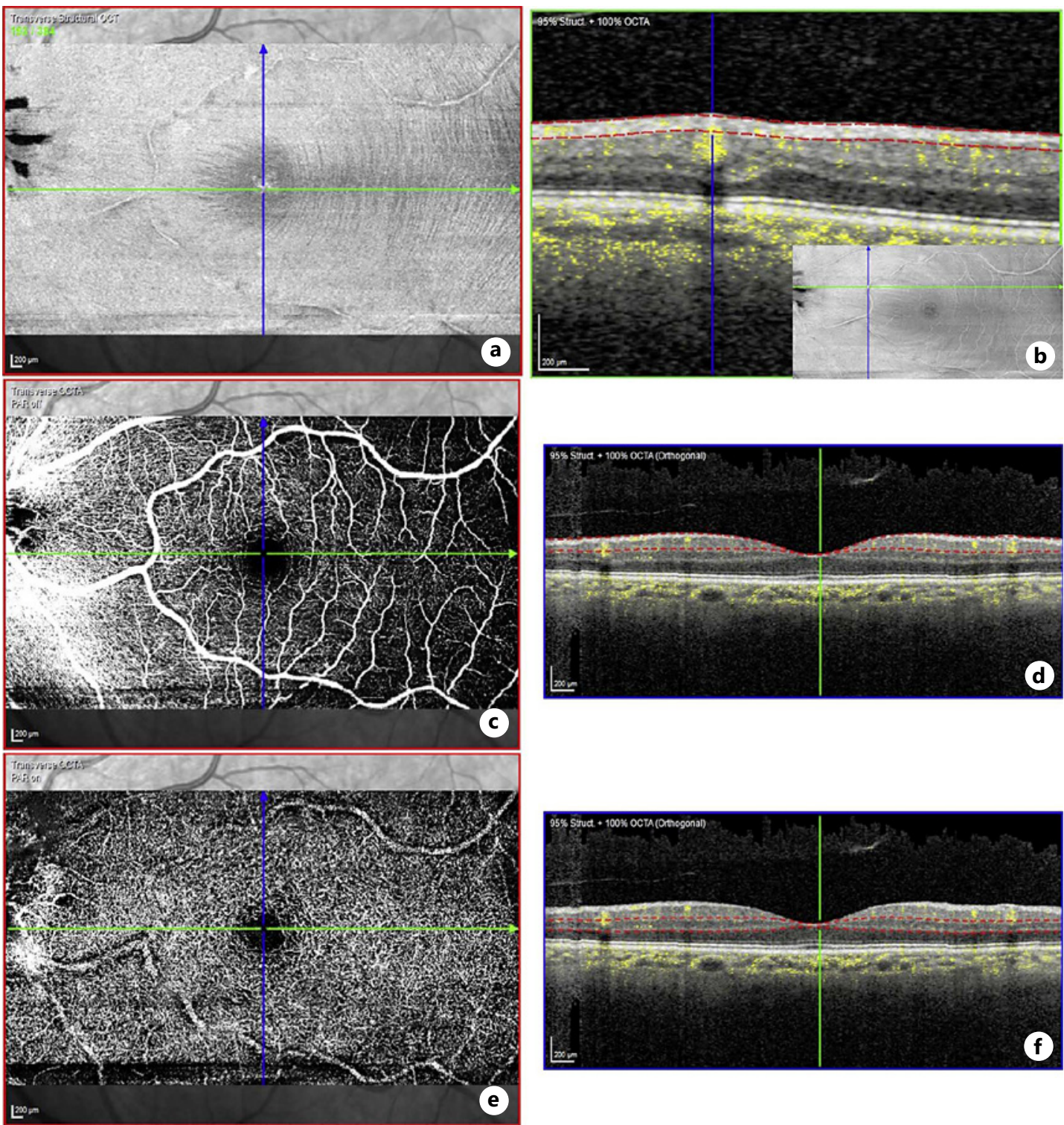

Fig. 1. A, C Multimodal imaging of the arterial vessel arising from the inferotemporal artery. SD-OCT imaging showing normal thickness of the peripapillary retinal nerve fiber layer (B) and normal macular morphology (D). En face (C, E) and OCTA (F-J) images showing the normal trajectory of the axons in the papillomacular bundle and the normal morphology of the horizontal raphe (E), the location of the vessel under the nerve fiber layer in the papillomacular bundle (F, inset), and the normal structure of the superficial $(\mathbf{G}, \mathbf{H})$ and deep (I, J) vascular plexuses.

only at the level of the deep capillary plexus $[2,3]$. However, at the temporal raphe, there is a distinct separation between the nerve fiber layer of the superior and inferior temporal retina, and this has been attributed to the earlier development of the central retina and to the existence of the 2 watershed zones [4]. The separate circulation of the superior and inferior hemispheres of the temporal retina might be a consequence of development and, in particular, of the organization of the long posterior ciliary arteries, and it has been suggested that it may also play a protective role in the case of vascular occlusive diseases [3]. 
Retinal vessel development initiates in the optic nerve and proceeds centripetally, respecting the temporal raphe and reaching the retinal periphery before birth [1]. In general, in the temporal retina, 2 retinal arteries, superior and inferior, develop that irrigate the superior and inferior macula, respectively. In 15-50\% of the individuals, however, the papillomacular or even a greater macular area is irrigated by arteries that arise from the posterior ciliary vessels, the so-called cilioretinal arteries [8]. In $<1 \%$ of the population, a large aberrant artery arises from the central retinal artery, from the superior or inferior arteries or from the temporal arteries to irrigate parts of the macula or bifurcate to irrigate the whole macula $[8,9]$. Our case belongs to this last category: an artery arises from the inferotemporal artery to irrigate the whole macula.

We present the incidental finding of a retinal artery that arises from the inferotemporal artery and divides into 2 branches: superior that crosses the papillomacular bundle perpendicularly and inferior to supply the superior and inferior macula. We wondered whether the vascular plexuses of the retina and the nerve fiber layer and raphe had developed normally in our patient, as this had not been investigated before in the rare cases reported of aberrant macular arteries, and it could be dependent on the existence of 2 watershed territories [4, 5, 8, 9]. We document, using standard and en face SD-OCT, that the vessel does not cause an alteration of the thickness of the peripapillary nerve fiber layer or the trajectory of the retinal ganglion cell axons, nor in the papillomacular bundle, because it lies under the fiber layer, nor in the temporal raphe. Macular structure was normal, and the SD-OCTA documented also a normal morphology of the retinal capillary plexuses, and we therefore did not perform a fluorescein angiography. In our case, the whole macula is irrigated by the same artery, and this documents that the formation of the temporal retinal nerve fiber raphe does not depend on the presence of 2 arterial territories in the retina. The retinal vasculature is the last in the eye to develop, and the foveal vasculature is not complete until just before birth [1]. The superficial (inner) vascular retinal plexus appears first and develops following molecular and functional clues provided by the retinal neurons and/or glial cells, and therefore it is believed that neurons/glial cells and vessels form a functional unit: the neurovascular unit [1, 10]. Although vessels and axon trajectories many times have parallel courses, sometimes as in our patient this is not true, and presumably this may be the case also in the retinal arterio-venous communications (retinal venous malformations or macrovessels) that cross the retinal raphe. The molecular and functional clues underlying these anatomical variations remain to be investigated.

\section{Summary Statement}

We report a very rare case of an anomalous retinal artery that originating from the inferotemporal artery irrigated the whole macula. Multimodal imaging, en face, and OCT angiography allowed us to document for the first time in this anatomical variation normal vascular retinal plexuses and retinal nerve fiber and raphe.

\section{Acknowledgment}

This manuscript does not include any nonauthor contributors to acknowledge.

\section{Statement of Ethics}

Written informed consent was obtained from the patient for publication of this case report and any accompanying images. This report does not require approval because this case is a casual finding. However, the patient signed an informed written consent and

\section{Karger's}


authorized the use of the images. This study protocol was reviewed, and the need for approval was waived by the Clinical Ethics Committee of the Hospital General Universitario Reina Sofia.

\section{Conflict of Interest Statement}

There are no financial conflicts of interest to disclose.

\section{Funding Sources}

We acknowledge the support received from Bloss Group and Heidelberg Engineering for the publication of this article.

\section{Author Contributions}

All authors have approved the final version of the manuscript. Santos-Bueso, VillegasPerez, and Garcia-Feijoo were involved in study concept and design. Vidal-Villegas, Miralles de Imperial-Ollero, Santos-Bueso, and Villegas-Perez were involved in acquisition, analysis, or interpretation of data. Vidal-Villegas, Miralles de Imperial-Ollero, and Villegas-Perez drafted the manuscript. Santos-Bueso, Villegas-Perez, and Garcia-Feijoo were involved in critical revision of the manuscript for important intellectual content. Vidal-Villegas, Miralles de Imperial-Ollero, Santos-Bueso, Villegas-Perez, and Garcia-Feijoo were involved in administrative, technical, or material support. Vidal-Villegas, Miralles de Imperial-Ollero, SantosBueso, Villegas-Perez, and Garcia-Feijoo were involved in study supervision.

\section{Data Availability Statement}

All data generated or analyzed during this study are included in this article and its online suppl. material files. Further enquiries can be directed to the corresponding author.

\section{References}

1 Lutty GA, McLeod DS. Development of the hyaloid, choroidal and retinal vasculatures in the fetal human eye. Prog Retin Eye Res. 2018;62:58-76.

2 Nesper PL, Fawzi AA. Human parafoveal capillary vascular anatomy and connectivity revealed by optical coherence tomography angiography. Invest Ophthalmol Vis Sci. 2018;59:3858-67.

3 May CA, Rutkowski P. The horizontal raphe of the human retina and its watershed zones. Vision. 2019;3:3-4.

4 Chauhan BC, Sharpe GP, Hutchison DM. Imaging of the temporal raphe with optical coherence tomography. Ophthalmology. 2014;121:2287-8.

5 Awan KJ. Anomalies of the retinal veins and their incidence. J Pediatr Ophthalmol. 1976;13:353-9.

6 Brown GC, Donoso LA, Magargal LE, Goldberg RE, Sarin LK. Congenital retinal macrovessels. Arch Ophthalmol. 1982;100:1430-6.

7 Pichi F, Freund KB, Ciardella A, Morara M, Abboud EB, Ghazi N, et al. Congenital retinal macrovessel and the association of retinal venous malformations with venous malformations of the brain. JAMA Ophthalmol. 2018; 136:372-9.

8 Awan KJ. Arterial vascular anomalies of the retina. Arch Ophthalmol. 1977; 95:1197-202.

9 Pipelart V, Bresson T, de Saint-Martin G. Retinal arterial variant: report of 2 cases. J Fr Ophtalmol. 2019;42: 213-4.

10 Biswas S, Cottarelli A, Agalliu D. Neuronal and glial regulation of CNS angiogenesis and barriergenesis. Development. 2020;147:dev182279.

\section{Karger's}

